\title{
Hypophosphatemia at Admission is Associated with Increased Mortality in COVID-I9 Patients
}

\author{
Ruoran Wang ${ }^{1, *}$ \\ Min $\mathrm{He}^{1,2, *}$ \\ Yan Kang (D) ${ }^{1,2}$ \\ 'Department of Critical Care Medicine, \\ West China Hospital, Sichuan University, \\ Chengdu, 6I004I, Sichuan Province, \\ People's Republic of China; ${ }^{2}$ COVIDI9 \\ Medical Team (Hubei) of West China \\ Hospital, Sichuan University, Chengdu, \\ People's Republic of China \\ *These authors contributed equally to \\ this work
}

Correspondence: Yan Kang

Department of Critical Care Medicine,

West China Hospital, No. 37, Guo Xue

Xiang, Chengdu, Sichuan Province,

People's Republic of China

Tel +862885422456

Fax +862885423453

Email kangyan@scu.edu.cn
Background: Electrolyte disturbances are commonly observed in patients with coronavirus disease 2019 (COVID-19) and associated with outcome in these patients. Our study was designed to examine whether hypophosphatemia is associated with mortality in COVID-19 patients.

Methods: Patients diagnosed with COVID-19 and hospitalized in Renmin Hospital of Wuhan University between January 30 and February 24, 2020 were included in this study. Patients were divided into two groups, a hypophosphatemia group and a nonhypophosphatemia group, based on a serum phosphate level of $0.8 \mathrm{mmol} / \mathrm{L}$. Logistic regression was performed to analyze the relationship between hypophosphatemia and mortality. A locally weighted scatterplot smoothing (LOWESS) curve was plotted to show the detailed association between mortality rate and serum phosphate level. A Kaplan-Meier survival curve was drawn to compare the difference in cumulative survival between the two groups.

Results: Hypophosphatemia at admission occurred in 33 patients, with an incidence of $7.6 \%$. The hypophosphatemia group had a significantly higher incidence of respiratory failure $(54.5 \%$ vs $32.6 \%, p=0.013)$ and mortality $(57.6 \%$ vs $15.2 \%, p<0.001)$. Multivariate logistic regression indicated that age $(\mathrm{OR}=1.059, p<0.001)$, oxygen saturation $(\mathrm{OR}=0.733$, $p<0.001)$, white blood cells ( $\mathrm{OR}=1.428, p<0.001)$, lymphocytes $(\mathrm{OR}=0.075, p<0.001)$ and hypophosphatemia $(\mathrm{OR}=3.636, p=0.015)$ were independently associated with mortality in the included patients. The hypophosphatemia group had significantly shorter survival than the non-hypophosphatemia group $(p<0.001)$.

Conclusion: Hypophosphatemia at admission is associated with increased mortality in COVID-19 patients. More attention and medical care should be given to COVID-19 patients with hypophosphatemia at admission.

Keywords: hypophosphatemia, serum phosphate, COVID-19, mortality

\section{Introduction}

Since the outbreak of coronavirus disease 2019 (COVID-19), more than 139 million people have been confirmed as having been infected and nearly 3 million infected individuals have died. As a severe public health issue, the COVID-19 pandemic has placed a massive burden on the global economy and medical resources. It has been demonstrated that dehydration and electrolyte disturbances commonly occur and may be associated with severity of the disease and the prognosis of COVID-19 patients. $^{1-3}$ Several studies have found that hyperkalemia, hypokalemia, hyponatremia and hypocalcemia were associated with increased mortality rates in COVID-19 patients. ${ }^{4-10}$ Compared with those 
who had normal levels of electrolytes, patients who developed electrolyte imbalances such as hyponatremia, hypokalemia, hypochloremia and hypocalcemia usually had more severe disease and were more likely to receive mechanical ventilation. ${ }^{2,-9,11-13}$ However, despite its being a widely observed electrolyte disturbance in hospitalized patients, hypophosphatemia was not investigated in COVID-19 patients. Previous studies have discovered that the incidence of hypophosphatemia ranged from $15.4 \%$ to $71.6 \%$ in a variety of patients, including critically ill children and adults, patients with acute kidney injury and those with massive burns. ${ }^{14-21}$ The correlation between increased mortality and occurrence of hypophosphatemia was also confirmed in these studies. We designed this study to explore the incidence of hypophosphatemia at admission in COVID-19 patients and to verify whether hypophosphatemia was associated with mortality in COVID-19 patients.

\section{Materials and Methods}

\section{Patients}

Patients with confirmed COVID-19 who were hospitalized in Renmin Hospital of Wuhan University between January 30 and February 24, 2020 were eligible for this study. Patients who did not have complete records of the required variables were excluded. The diagnosis of COVID-19 was based on positive results of COVID-19 RNA in samples of nasopharyngeal swabs acquired once patients were admitted to the hospital. The severity of disease in COVID-19 patients was judged based on the "Diagnosis and Treatment Protocol for Novel Coronavirus Infection-Induced Pneumonia version 8" in China. This guideline divides patients into four groups: mild type, common type, severe type and critical type. Our hospital gives priority to the treatment of severe and critical patients. When there are spare beds, non-severe patients can be received. Therefore, patients included in this study were mainly severe and critical patients.

\section{Data Collection}

Records of clinical and laboratory variables were collected from the electronic medical record (EMR) system of Renmin Hospital of Wuhan University. Comorbidities, including hypertension, chronic obstructive pulmonary disease (COPD), diabetes mellitus, chronic liver disease and malignancy, were recorded. Laboratory tests of the first blood sample, obtained when patients were admitted to hospital, were presented. In this study, patients were divided into two groups, the hypophosphatemia group and non-hypophosphatemia group, based on the criterion baseline serum phosphate level below $0.8 \mathrm{mmol} / \mathrm{L} .^{20}$ Hyperphosphatemia was generally confirmed when the serum phosphate level was above $1.6 \mathrm{mmol} / \mathrm{L}$. However, since only six included patients were confirmed to have hyperphosphatemia, we did not divide these patients into another hyperphosphatemia group but included them in the non-hypophosphatemia group. Only patients who had fully recovered from COVID-19 could be discharged from hospital. Therefore, the primary outcome of this study was inhospital mortality. Patients were followed up until 1 month after discharge.

This study obtained approval from the ethics committee of Renmin Hospital of Wuhan University and West China Hospital of Sichuan University. Informed consent of included COVID-19 patients was signed by the patients or their legal representatives. We designed and conducted this study according to the Declaration of Helsinki.

\section{Statistical Analysis}

The Kolmogorov-Smirnov test was performed to test the normality of variables. We presented normally distributed variables and non-normally distributed variables as mean \pm standard deviation and median (interquartile range), respectively. Categorical variables were presented in the form of counts (percentage). Student's $t$-test and Mann-Whitney $U$-test were, respectively, conducted to analyze the differences in normally distributed variables and nonnormally distributed variables between the hypophosphatemia group and non-hypophosphatemia group. To compare differences in categorical variables between the two groups, the chi-squared test or Fisher's exact test was performed. The Kaplan-Meier method was utilized to compare the difference in survival between the hypophosphatemia group and non-hypophosphatemia group. Univariate logistic regression was first performed to find potential risk factors for mortality in the included COVID-19 patients. Factors with statistical significance $(p<0.2)$ were then included in the multivariate logistic regression using the backward stepwise method. Odds ratios (ORs) and 95\% confidence intervals (Cls) of statistically significant risk factors were also presented. The detailed relationship between serum phosphate level and mortality rate was illustrated using a locally weighted scatterplot smoothing (LOWESS) curve, which is a powerful polynomial regression tool to view the nonlinear relationship between two-dimensional variables. 
Spearman's rank correlation was performed to analyze the relationship between the serum phosphate level and other variables.

A two-sided $p$-value $<0.05$ was considered to be of statistical significance. We utilized SPSS 22.0 for Windows software (IBM Corp., Armonk, NY, USA) and $\mathrm{R}$ (version 3.6.1; R Foundation) to perform statistical analyses and draw the figures.

\section{Results}

\section{Baseline Characteristics of Included COVID-19 Patients}

In total, 435 COVID-19 patients were included in this study and were divided into the hypophosphatemia group and non-hypophosphatemia group based on the serum phosphate level at admission. Hypophosphatemia at admission occurred in 33 patients, with an incidence of 7.6\% (Table 1). Compared with the non-hypophosphatemia group, the hypophosphatemia group had significantly higher age (67 vs 56 years, $p<0.001$ ). Hypertension was the most common comorbidity, with a rate of $18.6 \%$ in included patients. The hypophosphatemia group had a higher percentage of complicated chronic liver disease than the nonhypophosphatemia group $(12.1 \%$ vs $3.5 \%, p=0.039)$. Symptoms, including fever (77.7\%), cough (56.3\%), dyspnea $(34.4 \%)$ and fatigue $(33.3 \%)$, were all usually observed among the included patients. Dyspnea was more generally observed in the hypophosphatemia group than the non-hypophosphatemia group $(54.5 \%$ vs $32.6 \%, p=0.013)$. Compared with the non-hypophosphatemia group, the hypophosphatemia group had significantly higher levels of WBCs $(p=0.020)$, neutrophils $(p=0.003)$ and AST $(p=0.002)$, and lower levels of lymphocytes $(p<0.001)$, platelets $(p=0.017)$ and albumin $(p<0.001)$. Regarding the severity of disease in the included patients, the percentage of severe and critical patients was higher in the hypophosphatemia group. Moreover, the hypophosphatemia group had a significantly higher incidence of both respiratory failure during the hospitalization $(54.5 \%$ vs $32.6 \%$, $p=0.013)$ and mortality $(57.6 \%$ vs $15.2 \%, p<0.001)$. Both the length of hospital stay ( 8 vs 9 days, $p=0.028$ ) and course of the disease ( 16 vs 20 days, $p=0.046$ ) were shorter in the hypophosphatemia group. The cumulative survival until the end of follow-up was significantly shorter in the hypophosphatemia group than in the non-hypophosphatemia group $\left(\chi^{2}=49.177, p<0.001\right)$.

\section{Association Between Hypophosphatemia and Mortality in COVID-19 Patients}

Univariate logistic regression analysis showed that age, male gender, history of hypertension, COPD, oxygen saturation, WBCs, lymphocytes, platelets, albumin, AST, serum creatinine, severity and hypophosphatemia were potentially associated with mortality (Table 2). However, after adjusting for confounding effects, only age, oxygen saturation, WBCs, lymphocytes and hypophosphatemia were independently associated with mortality in multivariate logistic regression analysis.

The LOWESS smoothed fit curve visually presented the correlation between the serum phosphate level and mortality rate of the included COVID-19 patients (Figure 1). As shown, the mortality rate gradually increased with decreasing serum phosphate level, especially when serum phosphate was lower than 1.0 $\mathrm{mmol} / \mathrm{L}$. In addition, the survival of the nonhypophosphatemia group was significantly longer than that of the hypophosphatemia group $(p<0.001)$ (Figure 2).

\section{Correlation Between Serum Phosphate Level and Other Factors}

Spearman correlation analysis indicated that the serum phosphate level was negatively and moderately correlated with age ( $r=-0.319, p<0.001)$ (Figure 3A), and positively and moderately correlated with lymphocyte count $(r=0.343, p<0.001)$ (Figure 3B). Other factors did not show significant associations with serum phosphate level (Table 3).

\section{Discussion}

The incidence of hypophosphatemia at admission in the COVID patients included in this study was $7.6 \%$, which was lower than the incidence of hypophosphatemia previously reported in other kinds of critically ill patients. This difference could be attributable to the characteristics of COVID-19 itself and the severity of the disease in these patients. Among 141 mildly or moderately ill patients, only three of them had hypophosphatemia at admission, which significantly decreased the overall incidence of hypophosphatemia in the included patients. This result was consistent with the findings of one previous study, which indicated that low serum phosphorus could discriminate severe or critical from other COVID-19 patients with good specificity, and detect confirmed patients among all included individuals with an improved 
Table I Baseline Characteristics of Included Patients

\begin{tabular}{|c|c|c|c|c|}
\hline Variables & $\begin{array}{l}\text { Total Patients } \\
(n=435)\end{array}$ & $\begin{array}{l}\text { Non-Hypophosphatemia }(n=402 \text {, } \\
92.4 \%)\end{array}$ & $\begin{array}{l}\text { Hypophosphatemia }(n=33 \text {, } \\
7.6 \%)\end{array}$ & $p$ \\
\hline Age (years) & $57(4 \mid-68)$ & $56(39-67)$ & $67(63-76)$ & $<0.001$ \\
\hline Male & $200(46.0 \%)$ & 187 (46.5\%) & 13 (39.4\%) & 0.428 \\
\hline \multicolumn{5}{|l|}{ Comorbidity } \\
\hline Hypertension & 81 (I8.6\%) & 71 (17.7\%) & $10(30.3 \%)$ & 0.091 \\
\hline Diabetes mellitus & $26(6.0 \%)$ & $23(5.7 \%)$ & $3(9.1 \%)$ & 0.435 \\
\hline COPD & $24(5.5 \%)$ & $20(5.0 \%)$ & $4(12.1 \%)$ & 0.099 \\
\hline Chronic liver disease & $18(4.1 \%)$ & 14 (3.5\%) & $4(12.1 \%)$ & 0.039 \\
\hline Malignancy & $6(1.4 \%)$ & $4(1.0 \%)$ & $2(6.1 \%)$ & 0.069 \\
\hline \multicolumn{5}{|l|}{ Signs and symptoms } \\
\hline Fever & $338(77.7 \%)$ & $310(77.1 \%)$ & $28(84.8 \%)$ & 0.285 \\
\hline Cough & 245 (56.3\%) & $229(57.0 \%)$ & $16(48.5 \%)$ & 0.347 \\
\hline Dyspnea & 149 (34.3\%) & 131 (32.6\%) & 18 (54.5\%) & 0.013 \\
\hline Fatigue & 145 (33.3\%) & $133(33.1 \%)$ & $12(36.4 \%)$ & 0.703 \\
\hline Diarrhea & $34(7.8 \%)$ & $30(7.5 \%)$ & $4(12.1 \%)$ & 0.312 \\
\hline Oxygen saturation (\%) & $99.0(99.0-99.0)$ & $99.0(99.0-99.0)$ & $99.0(92.5-99.0)$ & \\
\hline \multicolumn{5}{|l|}{ Laboratory results } \\
\hline WBCs $\left(\times 10^{9} / \mathrm{L}\right)$ & $5.76(4.25-7.8 I)$ & $5.70(4.22-7.65)$ & $7.24(5.4 \mathrm{I}-8.64)$ & 0.020 \\
\hline Neutrophils $\left(\times 10^{9} / \mathrm{L}\right)$ & $3.79(2.59-5.98)$ & $3.66(2.55-5.59)$ & $6.09(3.77-6.63)$ & 0.003 \\
\hline Lymphocytes $\left(\times 10^{9} / \mathrm{L}\right)$ & $1.14(0.8 \mathrm{I}-1.56)$ & I.I8 (0.88-I.59) & $0.77(0.52-0.96)$ & $<0.001$ \\
\hline Monocytes $\left(\times 10^{9} / \mathrm{L}\right)$ & $0.44(0.30-0.61)$ & $0.45(0.32-0.61)$ & $0.37(0.26-0.50)$ & 0.061 \\
\hline Platelets $\left(\times 10^{9} / \mathrm{L}\right)$ & $215(158-273)$ & $217(161-278)$ & $180(|4|-23 \mid)$ & 0.017 \\
\hline Hemoglobin $(g / L)$ & $127(115-138)$ & $127(115-138)$ & $127(112-135)$ & 0.223 \\
\hline Albumin $(g / L)$ & $37.2 \pm 4.6$ & $37.4 \pm 4.5$ & $34.0 \pm 4.4$ & $<0.001$ \\
\hline Immunoglobulin $(g / L)$ & $24.1(21.7-27.5)$ & $24.0(21.7-27.4)$ & $24.9(22.4-28.4)$ & 0.391 \\
\hline ALT (U/L) & $25(16-39)$ & $25(16-39)$ & $26(18-39)$ & 0.478 \\
\hline AST (U/L) & $26(19-38)$ & $25(19-37)$ & $39(24-66)$ & 0.002 \\
\hline Serum creatinine $(\mu \mathrm{mol} / \mathrm{L})$ & $59(49-72)$ & $59(50-72)$ & $58(42-69)$ & 0.202 \\
\hline Phosphate (mmol/L) & I.II (0.96-I.25) & $1.13(0.99-1.28)$ & $0.73(0.64-0.78)$ & $<0.001$ \\
\hline Severity & & & & 0.008 \\
\hline Mild & $17(3.9 \%)$ & $16(4.0 \%)$ & I (3.0\%) & \\
\hline Moderate & 124 (28.5\%) & $122(30.3 \%)$ & $2(6.1 \%)$ & \\
\hline Severe & $160(36.8 \%)$ & $144(35.8 \%)$ & $16(48.5 \%)$ & \\
\hline Critical & $134(30.8 \%)$ & $120(29.9 \%)$ & $14(42.4 \%)$ & \\
\hline $\begin{array}{l}\text { Days from illness onset to admission } \\
\text { (days) }\end{array}$ & $10(7-13)$ & $10(7-13)$ & II (7-I2) & 0.803 \\
\hline \multicolumn{5}{|l|}{ Outcome } \\
\hline Length of hospital stay (days) & $9(5-14)$ & $9(5-14)$ & $8(3-11)$ & 0.028 \\
\hline Course of disease (days) & $20(15-26)$ & $20(15-27)$ & $16(14-22)$ & 0.046 \\
\hline Respiratory failure & 149 (34.3\%) & $|3|(32.6 \%)$ & I 8 (54.5\%) & 0.013 \\
\hline Mortality & 80 (I8.4\%) & $6 \mathrm{I}$ (I5.2\%) & 19 (57.6\%) & $<0.001$ \\
\hline
\end{tabular}

Abbreviations: COPD, chronic obstructive pulmonary disease; WBC, white blood cell; ALT, alanine aminotransferase; AST, aspartate aminotransferase.

area under the receiver operating characteristics curve (AUC). ${ }^{22}$ The results of other studies on COVID-19 also showed that confirmed patients had lower serum phosphorus levels than healthy controls, and severe or critical patients had lower phosphorus than moderate patients. ${ }^{23,24}$ In addition, the serum phosphorus level 
Table 2 Univariate and Multivariate Logistic Regression Analysis for the Association Between Hypophosphatemia and Mortality in Included COVID-19 Patients

\begin{tabular}{|c|c|c|c|c|c|c|}
\hline \multirow[t]{2}{*}{ Variables } & \multicolumn{3}{|c|}{ Unadjusted Analysis } & \multicolumn{3}{|c|}{ Adjusted Analysis } \\
\hline & OR & $95 \% \mathrm{Cl}$ & $p$ & OR & $95 \% \mathrm{Cl}$ & $p$ \\
\hline Age (years) & 1.088 & $1.064-1.112$ & $<0.001$ & 1.059 & $1.030-1.088$ & $<0.001$ \\
\hline Male & 1.659 & $1.017-2.705$ & 0.042 & & & \\
\hline Hypertension & 4.163 & $2.427-7.140$ & $<0.001$ & & & \\
\hline Diabetes mellitus & 1.696 & $0.688-4.182$ & 0.252 & & & \\
\hline COPD & 6.068 & $2.608-14.119$ & $<0.001$ & & & \\
\hline Chronic liver disease & 2.318 & $0.843-6.374$ & 0.103 & & & \\
\hline Malignancy & $4.57 \mid$ & $0.905-23.081$ & 0.066 & & & \\
\hline Oxygen saturation & 0.741 & $0.678-0.808$ & $<0.001$ & 0.733 & $0.645-0.834$ & $<0.001$ \\
\hline WBCs & 1.365 & $1.256-1.484$ & $<0.001$ & 1.428 & $1.277-1.597$ & $<0.001$ \\
\hline Neutrophils & 1.002 & $0.996-1.009$ & 0.496 & & & \\
\hline Lymphocytes & 0.037 & $0.016-0.083$ & $<0.001$ & 0.075 & $0.026-0.220$ & $<0.001$ \\
\hline Monocytes & 0.523 & $0.175-1.563$ & 0.246 & & & \\
\hline Platelets & 0.991 & $0.987-0.994$ & $<0.001$ & & & \\
\hline Hemoglobin & 1.005 & $0.990-1.019$ & 0.534 & & & \\
\hline Albumin & 0.766 & $0.713-0.824$ & $<0.001$ & & & \\
\hline Immunoglobulin & 1.031 & $0.985-1.079$ & 0.192 & & & \\
\hline ALT & 1.005 & $0.998-1.011$ & 0.163 & & & \\
\hline AST & 1.022 & $1.012-1.031$ & $<0.001$ & & & \\
\hline Serum creatinine & 1.011 & $1.005-1.017$ & 0.001 & & & \\
\hline Hypophosphatemia & 7.587 & $3.612-15.936$ & $<0.001$ & 3.636 & $1.285-10.288$ & 0.015 \\
\hline Severity & & & $<0.001$ & & & \\
\hline Mild & I & Reference & & & & \\
\hline Moderate & $5.38 \times 10^{7}$ & - & 0.999 & & & \\
\hline Severe & $4.86 \times 10^{8}$ & - & 0.998 & & & \\
\hline Critical & $6.63 \times 10^{8}$ & - & 0.998 & & & \\
\hline
\end{tabular}

Abbreviations: COPD, chronic obstructive pulmonary disease; WBC, white blood cell; ALT, alanine aminotransferase; AST, aspartate aminotransferase.

was lower in non-survivors than in survivors of COVID19 , although without reaching statistical significance. ${ }^{25,26}$

According to the conclusions of previous research, the main risk factors and underlying mechanisms responsible for hypophosphatemia are malnutrition, massive energy consumption, excessive losses from the intestinal tract and kidneys, and respiratory alkalosis. ${ }^{27-30}$ Firstly, inadequate dietary intake and increased protein and energy consumption could decrease the total storage of phosphorus in the body and therefore put the body at high risk of hypophosphatemia. It has been demonstrated that hypophosphatemia is associated with lower levels of protein intake, albumin, creatinine, hemoglobin, body mass index, fat tissue index and lean tissue index, which indicate poor nutritional status. ${ }^{16}$ One study even found that hypophosphatemia was associated with an $86.4 \%$ possibility of malnutrition in elderly hospitalized patients. ${ }^{31}$ In addition, systemic inflammation and cytokine storm in critically ill patients can consume large amounts of energy and stimulate catabolic metabolism. ${ }^{32-35}$ During severe acute respiratory syndrome coronavirus-2 (SARS-CoV-2) infection, the immune cells consume a lot of ATP to facilitate resistance to viral invasion and damage. ${ }^{36}$ As an essential component in the synthesis and regeneration of ATP, phosphorus will also be depleted without an abundant supply. Although phosphate can be released from bone reserves and muscle storage by the stimulation of released cytokines, excessive catabolism in the muscles will lead to the breakdown of muscle cells and the eventual release of intracellular phosphate into the extracellular space. ${ }^{37}$ This mechanism may explain the occurrence of rhabdomyolysis and the prevalence of muscle soreness and fatigue in COVID-19 patients. $^{38}$ Secondly, gastrointestinal and renal tissue may be damaged by the cytokine storm and overactivated immune inflammatory response, which could, in turn, impede the reabsorption of phosphate. ${ }^{39}$ The widespread intestinal dysfunction and acute kidney injury seen in COVID-19 patients may be another risk 


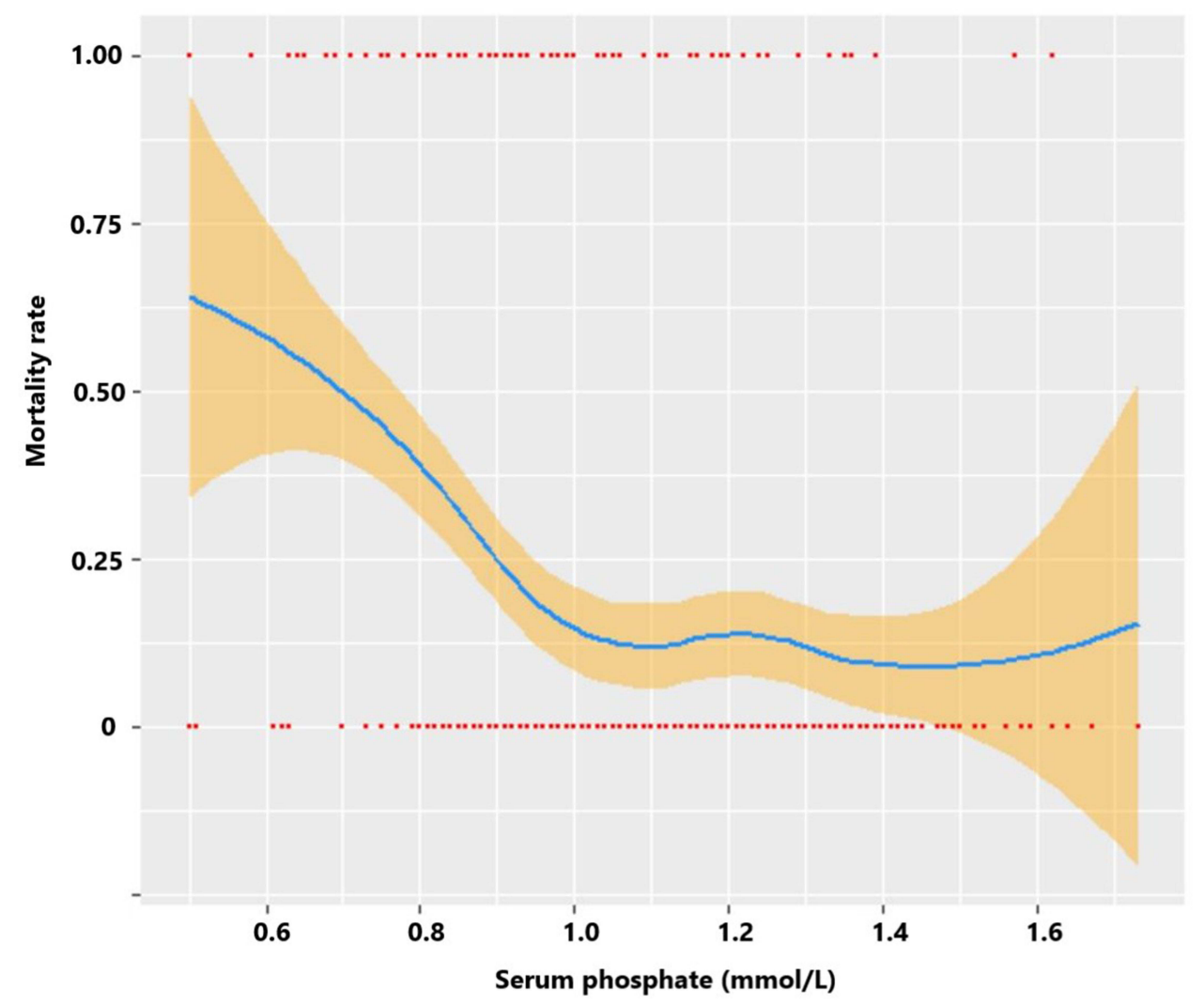

Figure I Relationship between serum phosphate level and mortality rate, using the LOWESS curve.

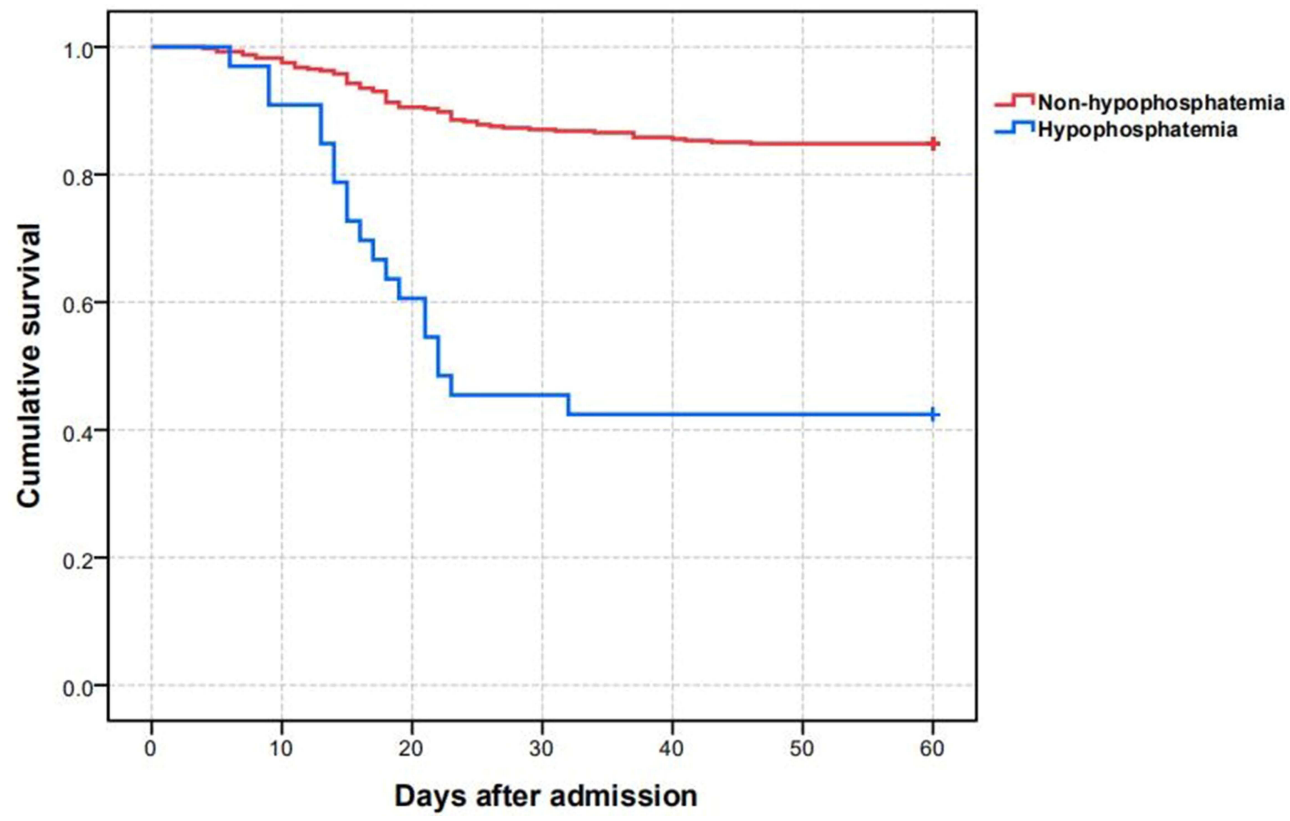

Figure 2 Differences in cumulative survival between hypophosphatemia group and non-hypophosphatemia group analyzed by the Kaplan-Meier method $\left(\chi^{2}=49.177\right.$, $p<0.001)$.

factor for hypophosphatemia. ${ }^{40-43}$ In addition, common digestive symptoms, such as diarrhea and vomiting, will also result in high levels of nutrient wastage. Thirdly, hyperventilation is another possible risk factor for hypophosphatemia. Respiratory alkalosis caused by hyperventilation is accompanied by increased intracellular $\mathrm{pH}$, 

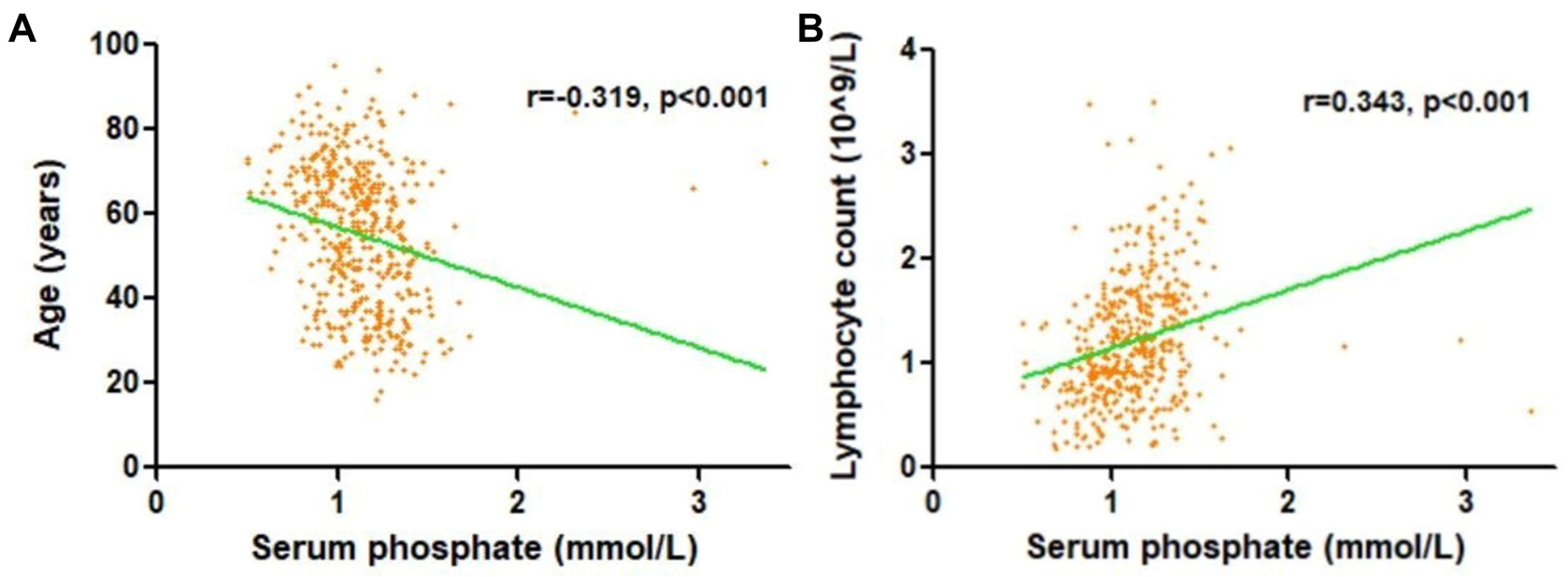

Figure 3 (A) Linear relationship between age and serum phosphate level. (B) Linear relationship between lymphocyte count and serum phosphate level.

which could upregulate the activity of phosphofructokinase and glycolysis. Phosphate would consequently be transferred from the extracellular space into the cytoplasm for this activity. ${ }^{30}$ Respiratory failure is a common complication among COVID-19 patients and many patients need respiratory support, with usage rates ranging from $14 \%$ to $81.7 \% .^{44-47}$ COVID-19 patients who develop respiratory failure usually undergo a process of

Table 3 Correlation Between Serum Phosphate and Other Factors

\begin{tabular}{|l|l|l|}
\hline Variables & $\boldsymbol{r}$ & $\boldsymbol{p}$ \\
\hline Age & $-\mathbf{0 . 3 1 9}$ & $<0.001$ \\
Male & -0.041 & 0.390 \\
Hypertension & -0.100 & 0.037 \\
Diabetes mellitus & -0.025 & 0.602 \\
COPD & -0.065 & 0.174 \\
Chronic liver disease & 0.016 & 0.737 \\
Malignancy & -0.033 & 0.490 \\
Oxygen saturation & 0.116 & 0.016 \\
WBCs & -0.061 & 0.206 \\
Neutrophils & -0.142 & 0.003 \\
Lymphocytes & 0.343 & $<0.001$ \\
Monocytes & 0.109 & 0.024 \\
Platelets & 0.230 & $<0.001$ \\
Hemoglobin & 0.106 & 0.027 \\
Albumin & 0.296 & $<0.001$ \\
Immunoglobulin & 0.001 & 0.978 \\
ALT & 0.002 & 0.960 \\
AST & -0.134 & 0.005 \\
Serum creatinine & 0.020 & 0.683 \\
Severity & -0.113 & 0.018 \\
\hline
\end{tabular}

Abbreviations: COPD, chronic obstructive pulmonary disease; WBC, white blood cell; ALT, alanine aminotransferase; AST, aspartate aminotransferase. compensatory hyperventilation with respiratory alkalosis before decompensation of respiration. ${ }^{48}$ This was confirmed in our study, where the hypophosphatemia group had a significantly higher incidence of respiratory failure during hospitalization.

Our study confirmed that hypophosphatemia was a risk factor for mortality in COVID-19 patients, with the hypophosphatemia group having a shorter cumulative survival than the non-hypophosphatemia group. The association between hypophosphatemia and poor outcome was probably mediated by the risk factors for hypophosphatemia mentioned in the previous paragraph. As a result of insufficient nutrient uptake and excessive energy consumption and catabolism, malnutrition was prevalent in COVID-19 patients and has been verified to be correlated with poor prognosis in these patients. ${ }^{49-54}$ In addition, the cellular ATP depletion, which may be reflected by the low serum phosphorus level, could influence the normal function of immune cells and impair the ability of the immune system to defend against attack by the virus. The correlation between immune dysfunction and hypophosphatemia was indicated by the positive and moderate association between lymphocyte count and serum phosphate level in our study. Furthermore, the hypophosphatemia attributable to losses from intestinal and renal tissue may indicate the severity of the cytokine storm and systemic inflammation among COVID-19 patients. And, undoubtedly, initially symptomized as hyperventilation with respiratory alkalosis, respiratory failure indicates a critical condition in COVID-19 patients and subsequent adverse progress.

Consequently, maintaining the homeostasis of phosphorus is necessary to prevent adverse progress in 
COVID-19 patients. Adequate supplementation with phosphate and other nutrients may decrease the probability of hypophosphatemia and improve the prognosis of these patients. There has been one study exploring the effect of combined supplements of vitamin $\mathrm{D}_{3}$, magnesium and vitamin $\mathrm{B}_{12}$ on the outcome of COVID-19 patients. ${ }^{55}$ Patients receiving combined supplements were less likely than controls to require oxygen support $(17.6 \%$ vs $61.5 \%)$ and intensive care unit support (5.9\% vs 30.8\%). Vitamin D plays a significant role in enhancing absorption from the intestinal tract and reducing kidney loss of phosphate and magnesium. One study on children with COVID-19 children also discovered that the vitamin D level was moderately associated with the serum phosphorus level $(r=0.364$, $p=0.020) .{ }^{23}$ Previous studies have confirmed the efficacy and safety of intravenous phosphate administration for correcting hypophosphatemia. ${ }^{56-60}$ One study demonstrated that extemporarily prepared phosphate buffer, administered via a nasogastric tube, could successfully correct the hypophosphatemia in non-surgical critically ill patients, without adverse effects. ${ }^{61}$ However, it is still unknown whether direct phosphate supplementation could effectively correct the hypophosphatemia and improve the prognosis of COVID-19 patients. In addition, the detailed dose and appropriate timing of phosphate administration in these patients is worth investigating in a future trial. In addition to medicinal supplements, available phosphate derived from dietary food intake and appropriate and timely respiratory management could avoid the development of hypophosphatemia, to some extent.

There were several limitations in this observational study. Firstly, this study was conducted in a single medical center, so selection bias could not be avoided. A further multicenter study with a larger sample size could be designed to verify our findings. Secondly, the occurrence of hyperphosphatemia at admission was scarce in the included COVID-19 patients, so we could not analyze the relationship between hyperphosphatemia and outcome in COVID-19 patients. Therefore, we incorporated these patients into the nonhypophosphatemia group. Thirdly, we only recorded the serum phosphate level obtained from tests of the first blood sample at admission. Hence, hypophosphatemia that developed during hospitalization could not be collected to analyze the relationship between subsequent low serum phosphate levels and mortality of the included patients. Future studies illustrating the tendency for serum phosphate fluctuation after admission would be worth performing in COVID-19 patients. Finally, the duration from illness onset to hospital admission in this study was 10 (7-13) days, which means that patients probably progress into the hyperinflammatory phase. However, the majority of COVID-19 patients in the present study did not have such a long duration from illness onset to hospital admission (usually less than 7 days). Thus, the hypophosphatemia at admission may not reliably reflect the initial status of infected patients and therefore the conclusions of this study should be interpreted with caution.

\section{Conclusions}

Although not a universally occurring electrolyte disturbance, hypophosphatemia is associated with increased mortality in COVID-19 patients. COVID-19 patients with hypophosphatemia at admission should be paid more attention to monitor the disease progression.

\section{Data Sharing Statement}

The data used and analyzed during this study are available from the corresponding author on reasonable request.

\section{Ethical Approval}

This study was approved by the ethics committee of West China Hospital of Sichuan University (2020 No.198) and Renmin Hospital of Wuhan University (WDRY2020K068). Informed consent of all included patients was obtained by us from the patients or their legal representatives. All the patient data used in the study were anonymized and de-identified. This study was conducted in accordance with the Declaration of Helsinki.

\section{Funding}

This research was supported by the Project of Novel Coronavirus Pneumonia in West China Hospital (project number: HX2019nCoV027).

\section{Disclosure}

All authors declare no conflicts of interest.

\section{References}

1. Lippi G, South AM, Henry BM. Electrolyte imbalances in patients with severe coronavirus disease 2019 (COVID-19). Ann Clin Biochem. 2020;57(3):262-265. doi:10.1177/0004563220922255

2. De Carvalho H, Richard MC, Chouihed T, et al. Electrolyte imbalance in COVID-19 patients admitted to the Emergency Department: a case-control study. Intern Emerg Med. 2021;1-6. doi:10.1007/ s11739-021-02632-z 
3. Bavaro DF, Diella L, Fabrizio C, et al. Peculiar clinical presentation of COVID-19 and predictors of mortality in the elderly: a multicentre retrospective cohort study. Int $J$ Infect Dis. 2021;105:709-715. doi:10.1016/j.ijid.2021.03.021

4. Liu J, Han P, Wu J, Gong J, Tian D. Prevalence and predictive value of hypocalcemia in severe COVID-19 patients. $J$ Infect Public Health. 2020;13(9):1224-1228. doi:10.1016/j.jiph.2020.05.029

5. Bennouar S, Cherif AB, Kessira A, Bennouar DE, Abdi S. Vitamin $\mathrm{D}$ deficiency and low serum calcium as predictors of poor prognosis in patients with severe COVID-19. J Am Coll Nutr. 2021;40 (2):104-110. doi:10.1080/07315724.2020.1856013

6. Liu S, Zhang L, Weng H, et al. Association between average plasma potassium levels and 30-day mortality during hospitalization in patients with COVID-19 in Wuhan, China. Int J Med Sci. 2021;18 (3):736-743. doi:10.7150/ijms.50965

7. Hu W, Lv X, Li C, et al. Disorders of sodium balance and its clinical implications in COVID-19 patients: a multicenter retrospective study. Intern Emerg Med. 2020:1-10. doi:10.1007/s11739-020-02515-9

8. De Carvalho H, Letellier T, Karakachoff M, et al. Hyponatremia is associated with poor outcome in COVID-19. J Nephrol. 2021:1-8. doi:10.1007/s40620-021-01036-8

9. Ruiz-Sánchez JG, Núñez-Gil IJ, Cuesta M, et al. Prognostic impact of hyponatremia and hypernatremia in COVID-19 pneumonia. A HOPE-COVID-19 (health outcome predictive evaluation for COVID-19) registry analysis. Front Endocrinol. 2020;11:599255. doi:10.3389/fendo.2020.599255

10. Alfano G, Ferrari A, Fontana F, et al. Hypokalemia in patients with COVID-19. Clin Exp Nephrol. 2021;25(4):401-409. doi:10.1007/ s10157-020-01996-4

11. Moreno PO, Leon-Ramirez JM, Fuertes-Kenneally L, et al. Hypokalemia as a sensitive biomarker of disease severity and the requirement for invasive mechanical ventilation requirement in COVID-19 pneumonia: a case series of 306 Mediterranean patients. Int $J$ Infect Dis. 2020;100:449-454. doi:10.1016/j. ijid.2020.09.033

12. Chen D, Li X, Song Q, et al. Assessment of hypokalemia and clinical characteristics in patients with coronavirus disease 2019 in Wenzhou, China. JAMA Netw Open. 2020;3(6):e2011122. doi:10.1001/ jamanetworkopen.2020.11122

13. Tezcan ME, Dogan Gokce G, Sen N, Zorlutuna Kaymak N, Ozer RS. Baseline electrolyte abnormalities would be related to poor prognosis in hospitalized coronavirus disease 2019 patients. New Microbes New Infect. 2020;37:100753. doi:10.1016/j.nmni.2020.100753

14. Santana e Meneses JF, Leite HP, de Carvalho WB, Lopes E Jr. Hypophosphatemia in critically ill children: prevalence and associated risk factors. Pediatr Crit Care Med. 2009;10(2):234-238. doi:10.1097/PCC.0b013e3181937042

15. Yang Y, Zhang P, Cui Y, et al. Hypophosphatemia during continuous veno-venous hemofiltration is associated with mortality in critically ill patients with acute kidney injury. Crit Care. 2013;17(5):R205. doi:10.1186/cc12900

16. Garagarza C, Valente A, Caetano C, Oliveira T, Ponce P, Silva AP. Hypophosphatemia: nutritional status, body composition, and mortality in hemodialysis patients. Int Urol Nephrol. 2017;49 (7):1243-1250. doi:10.1007/s11255-017-1558-2

17. Federspiel CK, Itenov TS, Thormar K, Liu KD, Bestle MH. Hypophosphatemia and duration of respiratory failure and mortality in critically ill patients. Acta Anaesthesiol Scand. 2018;62 (8):1098-1104. doi:10.1111/aas.13136

18. Yang HT, Yim H, Cho YS, et al. Change of serum phosphate level and clinical outcome of hypophosphatemia in massive burn patient. J Trauma Acute Care Surg. 2012;73(5):1298-1302. doi:10.1097/ TA.0b013e3182701e09
19. Shah SK, Irshad M, Gupta N, Kabra SK, Lodha R. Hypophosphatemia in critically ill children: risk factors, outcome and mechanism. Indian $J$ Pediatr. 2016;83(12-13):1379-1385. doi:10.1007/s12098-016-2188-x

20. Wang L, Xiao C, Chen L, Zhang X, Kou Q. Impact of hypophosphatemia on outcome of patients in intensive care unit: a retrospective cohort study. BMC Anesthesiol. 2019;19(1):86. doi:10.1186/s12871-019-0746-2

21. Berger MM, Appelberg O, Reintam-Blaser A, et al. Prevalence of hypophosphatemia in the ICU - results of an international one-day point prevalence survey. Clin Nutr. 2021;40(5):3615-3621.

22. Yang C, Ma X, Wu J, et al. Low serum calcium and phosphorus and their clinical performance in detecting COVID-19 patients. $J$ Med Virol. 2021;93(3):1639-1651. doi:10.1002/jmv.26515

23. Yılmaz K, SSen V. Is vitamin D deficiency a risk factor for COVID-19 in children? Pediatr Pulmonol. 2020;55(12):3595-3601. doi:10.1002/ ppul.25106

24. Pal R, Ram S, Zohmangaihi D, et al. High prevalence of hypocalcemia in non-severe COVID-19 patients: a Retrospective Case-Control Study. Front Med. 2020;7:590805. doi:10.3389/ fmed.2020.590805

25. Min Y, Cheng L, Tu C, et al. Clinical characteristics of deceased hemodialysis patients affected by COVID-19. Int Urol Nephrol. 2021;53(4):797-802. doi:10.1007/s11255-020-02700-x

26. Ghazanfari T, Salehi MR, Namaki S, et al. Interpretation of hematological, biochemical, and immunological findings of COVID-19 disease: biomarkers associated with severity and mortality. Iran J Allergy Asthma Immunol. 2021;20(1):46-66.

27. Sabbagh Y, Giral H, Caldas Y, Levi M, Schiavi SC. Intestinal phosphate transport. Adv Chronic Kidney Dis. 2011;18(2):85-90. doi:10.1053/j.ackd.2010.11.004

28. Levi M, Gratton E, Forster IC, et al. Mechanisms of phosphate transport. Nat Rev Nephrol. 2019;15(8):482-500.

29. Biber J, Hernando N, Forster I. Phosphate transporters and their function. Annu Rev Physiol. 2013;75:535-550. doi:10.1146/annurevphysiol-030212-183748

30. Datta BN, Stone MD. Hyperventilation and hypophosphataemia. Ann Clin Biochem. 2009;46(Pt 2):170-171. doi:10.1258/acb.2008.008199

31. Pourhassan M, Müller MJ, Volkert D, Wirth R. Hypophosphatemia as a sign of malnutrition in older hospitalized patients. Eur J Clin Nutr. 2019;73(4):634-636. doi:10.1038/s41430-018-0251-6

32. Deger SM, Hung AM, Gamboa JL, et al. Systemic inflammation is associated with exaggerated skeletal muscle protein catabolism in maintenance hemodialysis patients. JCI Insight. 2017;2(22). doi:10.1172/jci.insight.95185

33. Rong YD, Bian AL, Hu HY, Ma Y, Zhou XZ. Study on relationship between elderly sarcopenia and inflammatory cytokine IL-6, anti-inflammatory cytokine IL-10. BMC Geriatr. 2018;18(1):308. doi:10.1186/s12877-018-1007-9

34. Bistrian BR. Role of the systemic inflammatory response syndrome in the development of protein-calorie malnutrition in ESRD. Am J Kidney Dis. 1998;32(6 Suppl 4):S113-S117. doi:10.1016/S02726386(98)70173-8

35. Uribarri J, El Shamy O, Sharma S, Winston J. COVID-19-associated acute kidney injury and quantified protein catabolic rate: a likely effect of cytokine storm on muscle protein breakdown. Kidney Med. 2021;3(1):60-63.e61. doi:10.1016/j.xkme.2020.09.011

36. van Kempen T, Deixler E. SARS-CoV-2: influence of phosphate and magnesium, moderated by vitamin D, on energy (ATP) metabolism and on severity of COVID-19. Am J Physiol Endocrinol Metab. 2021;320(1):E2-E6. doi:10.1152/ajpendo.00474.2020

37. van Niekerk G, Mitchell M, Engelbrecht A-M. Bone resorption: supporting immunometabolism. Biol Lett. 2018;14(2):20170783. doi:10.1098/rsbl.2017.0783 
38. Mukherjee A, Ghosh R, Aftab G. Rhabdomyolysis in a patient with coronavirus disease 2019. Cureus. 2020;12(7):e8956.

39. Bülow Anderberg S, Luther T, Berglund M, et al. Increased levels of plasma cytokines and correlations to organ failure and 30-day mortality in critically ill Covid-19 patients. Cytokine. 2021;138:155389. doi:10.1016/j.cyto.2020.155389

40. Ghosn M, Attallah N, Badr M, et al. Severe acute kidney injury in critically ill patients with COVID-19 admitted to ICU: incidence, risk factors, and outcomes. J Clin Med. 2021;10(6):1217. doi:10.3390/ jcm 10061217

41. Hardenberg JB, Stockmann H, Aigner A, et al. Critical illness and systemic inflammation are key risk factors of severe acute kidney injury in patients with COVID-19. Kidney Int Rep. 2021;6 (4):905-915. doi:10.1016/j.ekir.2021.01.011

42. Allan PJ, Ambrose T, Mountford C, et al. COVID-19 infection in patients with intestinal failure: U.K. experience. J Parenter Enteral Nutr. 2021. doi:10.1002/jpen.2087

43. Zhang J, Garrett S, Sun J. Gastrointestinal symptoms, pathophysiology, and treatment in COVID-19. Genes Dis. 2021;8(4):385-400.

44. Grasselli G, Zangrillo A, Zanella A, et al. Baseline characteristics and outcomes of 1591 patients infected with SARS-CoV-2 admitted to ICUs of the Lombardy Region, Italy. JAMA. 2020;323 (16):1574-1581. doi:10.1001/jama.2020.5394

45. Wang D, Hu B, Hu C, et al. Clinical characteristics of 138 hospitalized patients with 2019 novel coronavirus-infected pneumonia in Wuhan, China. JAMA. 2020;323(11):1061-1069. doi:10.1001/jama. 2020.1585

46. Chen N, Zhou M, Dong X, et al. Epidemiological and clinical characteristics of 99 cases of 2019 novel coronavirus pneumonia in Wuhan, China: a descriptive study. Lancet. 2020;395(10223): 507-513. doi:10.1016/S0140-6736(20)30211-7

47. Cinesi Gómez C, Peñuelas Rodríguez Ó, Luján Torné M, et al. Clinical consensus recommendations regarding non-invasive respiratory support in the adult patient with acute respiratory failure secondary to SARS-CoV-2 infection. Med Intensiva. 2020;44 (7):429-438. doi:10.1016/j.medin.2020.03.005

48. Ottestad W, Søvik S. COVID-19 patients with respiratory failure: what can we learn from aviation medicine? Br J Anaesth. 2020;125 (3):e280-e281. doi:10.1016/j.bja.2020.04.012

49. Li T, Zhang Y, Gong C, et al. Prevalence of malnutrition and analysis of related factors in elderly patients with COVID-19 in Wuhan, China. Eur J Clin Nutr. 2020;74(6):871-875. doi:10.1038/s41430020-0642-3

50. Di Filippo L, De Lorenzo R, D’Amico M, et al. COVID-19 is associated with clinically significant weight loss and risk of malnutrition, independent of hospitalisation: a post-hoc analysis of a prospective cohort study. Clin Nutr. 2021;40(4):2420-2426. doi:10.1016/j.clnu.2020.10.043
51. Yu Y, Ye J, Chen M, et al. Malnutrition prolongs the hospitalization of patients with COVID-19 infection: a clinical epidemiological analysis. J Nutr Health Aging. 2021;25(3):369-373. doi:10.1007/ s12603-020-1541-y

52. Liu A, Cong J, Wang Q, et al. Risk of malnutrition is common in patients with Coronavirus Disease 2019 (COVID-19) in Wuhan, China: a Cross-sectional Study. J Nutr. 2021;151(6):1591-1596.

53. Wei C, Liu Y, Li Y, Zhang Y, Zhong M, Meng X. Evaluation of the nutritional status in patients with COVID-19. J Clin Biochem Nutr. 2020;67(2):116-121. doi:10.3164/jcbn.20-91

54. Zhou J, Ma Y, Liu Y, et al. A correlation analysis between the nutritional status and prognosis of COVID-19 patients. J Nutr Health Aging. 2021;25(1):84-93. doi:10.1007/s12603-020-1457-6

55. Tan CW, Ho LP, Kalimuddin S, et al. Cohort study to evaluate the effect of vitamin $\mathrm{D}$, magnesium, and vitamin $\mathrm{B}(12)$ in combination on progression to severe outcomes in older patients with coronavirus (COVID-19). Nutrition. 2020;79-80:111017. doi:10.1016/j.nut.2020.111017

56. Geerse DA, Bindels AJ, Kuiper MA, Roos AN, Spronk PE, Schultz MJ. Treatment of hypophosphatemia in the intensive care unit: a review. Crit Care. 2010;14(4):R147. doi:10.1186/cc9215

57. Rosen GH, Boullata JI, O'Rangers EA, Enow NB, Shin B. Intravenous phosphate repletion regimen for critically ill patients with moderate hypophosphatemia. Crit Care Med. 1995;23 (7):1204-1210. doi:10.1097/00003246-199507000-00009

58. Charron T, Bernard F, Skrobik Y, Simoneau N, Gagnon N, Leblanc M. Intravenous phosphate in the intensive care unit: more aggressive repletion regimens for moderate and severe hypophosphatemia. Intensive Care Med. 2003;29(8):1273-1278. doi:10.1007/s00134-003-1872-2

59. Taylor BE, Huey WY, Buchman TG, Boyle WA, Coopersmith CM. Treatment of hypophosphatemia using a protocol based on patient weight and serum phosphorus level in a surgical intensive care unit. J Am Coll Surg. 2004;198(2):198-204. doi:10.1016/j.jamcollsurg.2003.09.013

60. Brown KA, Dickerson RN, Morgan LM, Alexander KH, Minard G, Brown RO. A new graduated dosing regimen for phosphorus replacement in patients receiving nutrition support. J Parenter Enteral Nutr. 2006;30(3):209-214. doi:10.1177/0148607106030003209

61. Kovačević T, Kovačević P, Tomić B, Dragić S, Momčičević D. An alternative approach to treatment of hypophosphatemia in nonsurgical critically ill patients in countries with limited resources. Dose-Response. 2019;17(2):1559325819850421. doi:10.1177/1559325819850421
International Journal of General Medicine

\section{Publish your work in this journal}

The International Journal of General Medicine is an international, peer-reviewed open-access journal that focuses on general and internal medicine, pathogenesis, epidemiology, diagnosis, monitoring and treatment protocols. The journal is characterized by the rapid reporting of reviews, original research and clinical studies across all disease areas. The manuscript management system is completely online and includes a very quick and fair peer-review system, which is all easy to use. Visit http://www.dovepress.com/ testimonials.php to read real quotes from published authors. 